that such mischievous doctrines should be suppressed by the secular arm.

Finally, is it not time that this old bogy of the case of Galileo, as a proof that the Church was opposed to scientific research, should be decently buried? "The Papal power," wrote De Morgan (loc. cit.), " must on the whole have been moderately used in matters of philosophy, if we may judge by the great stress laid on this one case of Galileo." Cardinal Newman dubbed it rightly as "the one stock argument." A. L. Cortie, S.J. Stonyhurst College Observatory, Lancs. July 17.

\section{Surface Tension and Cell-Division.}

IN a recent paper on "Surface Tension and CellDivision" (Q.J.M.S. 66, pp. 235-245), Mr. J. Gray maintains that " cell-division may be accounted for by the movement of the two asters away from each other," and that " there is no necessity to postulate regions of differential surface tension at the poles or equator of the cell."

These conclusions are drawn chiefly from the results of a series of experiments in which the effect of "acid" sea-water on the cleaving eggs of Echinus miliaris is interpreted in the light of the effect of increased hydrogen-ion concentration on an oil-water interface. Mr. Gray points out that there is undoubtedly a lipoid or oily phase in the protoplasmic surface, but, if any value is to be placed on the analogy between the effect of acid on cleaving eggs and on an oil-water surface, one has to suppose that the lipoid phase in the protoplasmic surface is a continuous phase, otherwise two different types of surface are being dealt with. This view, as pointed out by Bayliss (2nd Brit. Assoc. Rep. Colloids, I9I 8 ), would necessitate that the volume of the cell should not have any definite relation to the osmotic pressure of the external solution.

In these experiments it was shown that if the egg, in which a cleavage furrow had appeared, is placed in "acid " sea-water, the furrow disappears and, owing to the unknown force which elongates the egg, equilibrium is attained when the egg. has the form of a cylinder with hemispherical ends. The shape of the dividing egg is stated to be dependent entirely on $(a)$ the surface tension at the egg surface, and $(b)$ the other force acting against the surface tension which produces elongation of the cell axis and is " associated in some way with the elongation of the astral figure." Now the pressure inside the fluid egg at any region due to surface tension can be determined from the curvature of the egg surface in that region. Since the egg is presumed to be in equilibrium at any stage during mitosis, any inequalities in pressure due to the surface tension and resulting from the varying curvature, must be counteracted by the second "elongating" force. In the case quoted above, in which the dividing egg is in equilibrium when its shape is that of a cylinder with hemispherical ends, it is easy to show that the pressure inside the hemispherical caps due to surface tension is exactly twice the pressure inside the cylindrical portion due to surface tension. Thus the elongating force, of which the astral figure is to some degree an expression, must produce a differential pressure on the inside of the egg surface, such that the pressure exerted over the middle cylindrical portion is uniform and equal to half the pressure produced over the hemispherical ends. If the view is accepted that the mitotic figure is in any way connected with the force that produces elongation and cleavage of the egg, I think the hypothesis that this force is essentially a polar force must be adopted, and it is difficult to see how any polar force could produce such a distribution of forces as is necessitated to produce equilibrium in the fluid cylindrical egg described by Mr. Gray.

$\mathrm{He}$ further describes an experiment as due to Plateau, on a drop of oil placed between two metal rings "so as to form a complete cylinder" (of course, with spherical end surfaces), which, on moving the two rings apart, changed in form, when the distance between the rings became greater than $\frac{2}{3}$ the diameter of the rings, in the same way as a dividing egg. The exact reference to this experiment is not given, but the experiment is very similar to one that Plateau carried out on the stability of a fluid catenoidal surface. According to Mr. Gray a cleaving egg can be divided into three parts : a middle cylindrical portion and two convex ends to this cylinder. The middle cylinder is taken to be analogous to the drop of oil in Plateau's experiment. It is obvious, however, that the whole of this experiment depends on the fact that the two rings, as they are being moved apart, exert a lateral thrust on the surface film of the drop. Is one, then, to believe that the force which causes the egg to elongate also exerts a lateral thrust in two parallel planes corresponding to the two rings? Actually, if there is sufficient oil between the two rings they may be moved apart to a distance equal to $\pi$ times the diameter of the rings before the central cylindrical portion becomes unstable and divides, but this in no way resembles the form of any cleaving egg, so far as I am aware.

While I quite agree with Mr. Gray that the mitotic force counteracts the effects due to surface tension, it seems to me that one cannot account for the stability of his cylindrical stage on the supposition of uniform surface tension over the whole egg surface, without ascribing. to that mitotic force properties that one would hesitate to ascribe to forces focussed round two definite centres. Unless the view that the forces which elongate the egg are correlated with the spatial relations of the two centrosomes is adopted, all the morphological evidence of the mechanism of cell-division is neglected.

In the introduction to his paper Mr. Gray says that theories postulating a higher or lower surface tension at the equator of a cleaving cell are of little value, as "there is no apparent means of determining how such a state of affairs could arise." In many cells it has been shown that the cytoplasmic inclusions are arranged around the centrosomes as foci, and further, that the distribution of these elements may be correlated with the apparent activity of the centrosomes. From this it may be inferred that there are substances in the egg which are repelled or attracted, either directly or indirectly, by the centrosomes. One of the first events in the cleavage of an Echinus egg is the appearance of two " active " centrosomes. These are approximately symmetrically placed in the egg and move apart from one another. Substances repelled by the centrosomes will tend to gather at the surface of the egg, but, as the centrosomes move apart, these bodies, once having reached the surface, will move towards that region of the egg surface in the plane in between the two centrosomes, which is termed the equator of the egg. If the presence of these substances in the surface film of the egg affects in any way the surface tension of the protoplasmic surface, here is a mechanism whereby there may arise a differential surface tension over the surface of the cleaving egg. H. GRAHAM CANNON.

I mperial College of Science and Technology, South Kensington, London, S.W.7,

$$
\text { July I4. }
$$

